\title{
Evaluation of copper and haematological markers in atherosclerosis coronary
}

\section{heart disease}

\author{
Preeti Gupta1 ${ }^{1}$ P.B. Desai ${ }^{2}$
}

1. L.N. Medical College \& RC, Bhopal, Madhya Pradesh, India

2. D M Wayanad Institute of Medical Sciences, Wayanad, Kerela, India

\section{RESEARCH}

Please cite this paper as: Gupta P, Desai PB. Evaluation of copper and haematological markers in atherosclerosis coronary heart disease. AMJ 2020;13(5):156-162.

https://doi.org/10.35841/1836-1935.13.5.156-162

\section{Corresponding Author: \\ Preeti Gupta \\ Associate Professor \\ Department of Biochemistry \\ L.N. Medical College \& Research Center \\ Bhopal-462042, Madhya Pradesh, India \\ Email: drpreetigs@gmail.com}

\section{ABSTRACT}

\section{Background}

Coronary heart disease [CHD] remains the major cause of morbidity and mortality in all developed as well as in developing countries. Leading cause of $\mathrm{CHD}$ is atherosclerosis, an inflammatory disease. Inflammation is closely related to thrombosis which begins with Platelets aggregation. Experimental Studies on animal model had been highlighted that copper may be associated with platelet aggregation but the data in human studies are lacking.

\section{Aims}

This study was undertaken to evaluate role of haematological markers namely platelet count, clotting time, bleeding time and association of copper in pathogenesis of CHD.

\section{Methods}

The study was conducted was conducted in a single centre with retrospective atherosclerotic CHD cases and 100 as healthy controls. All 200 subjects were undergone through
ECG and invasive coronary angiography. Haematological markers such as platelet counts, clotting time, bleeding time \& Copper were measured in both groups by standard biochemical methods.

\section{Results}

Patients with the atherosclerotic CHD had decreased level of Platelet counts \& Copper compared to healthy controls whereas there were no significant difference had been observed in CT \& BT. Significant Positive correlation observed among the platelet count and copper levels in CHD cases. Post hoc analysis of platelet count and copper level in CHD cases and control subjects shows, significant positive correlation between the copper and platelet count levels in CHD cases, although on contrary there was nonsignificant negative correlation observed in copper and platelet counts levels among the control subjects.

\section{Conclusion}

Compared to control, low platelet count and copper levels in cases predict that platelets and copper may plays a crucial role in the development of atherosclerosis CHD and has correlation with each.

\section{Key Words}

Atherosclerosis, platelets, copper, coronary heart disease

\section{What this study adds:}

\section{What is known about this subject?}

Pathogenesis of Atherosclerosis is not only correlates with accumulation of lipid but it is an inflammatory disease associated with thrombosis of the injured vascular wall.

\section{What new information is offered in this study?} Serum copper levels could be consider as diagnostic indicators of atherosclerosis as it is found to significantly correlated with platelet concentration.

3. What are the implications for research, policy, or practice?

Attention should be paid to the trace elements along with 
other markers for the diagnosis of atherosclerotic CHD.

\section{Background}

Cardiovascular disease (CVD) is accountable for approximate 30 percent of all death worldwide per annum and it is worth mentioning that approximately 80 percent of these casualties occur in low and middle income countries. ${ }^{1}$ Thus, it is a considerable health concern. 150,000 to $450,000 /$ platelets are found in per millilitre of healthy individual's blood to address the issue of bleeding during injury by thrombosis. ${ }^{2}$ Moreover, platelet aggregation is associated with atherosclerotic CHD events therefore it generally addressed by giving anti-platelet aggregation therapy as a preventive medication in high risk individuals. Although, the role of platelets in the atherogenesis of $\mathrm{CHD}$ is well recognized, there is contradictory evidence from observational studies on the relationship between platelet count and risk of atherosclerotic CHD. Better understanding of this association would bring more close to the pathophysiology of atherosclerosis, which may sequentially guide towards disease prevention.

Based on the animal experimental model which suggests that copper deficiency leads to platelet aggregation via decreased VWF and ADP. ${ }^{3}$ Therefore, this study was designed to evaluate the impact of haematological markers like Platelet counts, Clotting and Bleeding time and its association with copper in atherosclerotic coronary heart disease.

\section{Methods}

This Cross- sectional study was conducted at Department of Biochemistry, Department of Pathology J. N. Medical College and Heart Foundation of K.L.E.S. Dr. Prabhakar Kore Hospital and Medical Research Centre, Belgaum (Karnataka) from January 2009 to October 2010. Patients included in the present study were all admitted to the Intensive Coronary Care Unit (ICCU) or Department of Cardiology/ Heart Foundation of KLE Dr. Prabhakar Kore Hospital and MRC attached to Jawaharlal Nehru Medical College Belgaum (Karnataka). 100 subjects aged from 40-65 years who admitted in the hospital after clinically diagnosis of Atherosclerotic Coronary Heart Disease, were recruited for the study. The criteria for the diagnosis of atherosclerotic CHD was made on the basis of clinical history, 12 leads electrocardiogram (ECG) and invasive coronary angiography based on $\mathrm{X}$ ray findings. The clinically suspected patient of CHD were screened by ECG and further confirmed through invasive coronary angiography were recruited as cases for the study. All patients were having Atherosclerotic CHD but non Congenital Heart Disease \& Infective Endocarditis, diabetes mellitus, tuberculosis and renal function disorders as well as having bleeding disorders, and those on anticoagulant therapy. The 100, apparently healthy age and sex matched subjects, not having any clinical and ECG changes as symptoms of CHD. Such apparently healthy subjects were asked to participate as control in this study. Controls were also undergone by invasive coronary angiography to confirm no atherosclerotic CHD.

All participants gave written consent and this protocol was approved by the ethical and research committee of Jawaharlal Nehru Medical College, Belgaum.

Venous blood samples were collected from all the study subjects. Estimation of clotting time was based on Lee White method ${ }^{4}$ and estimation of bleeding time was based on Duke's Method ${ }^{5}$ and platelet counts was based on the Rees-Ecker Method ${ }^{6}$ whereas estimation of copper by Atomic Absorption Spectrophotometer respectively.

\section{Sample size calculations}

Sample size was calculated as 200 subjects for this study. The sample size was calculated to estimate mean values of parameters. The formula used was $4 \mathrm{SD}^{2} / \mathrm{d}^{2}$.

One hundred atherosclerotic CHD cases and equal number of apparently healthy controls matched for age and sex were included in the study.

\section{Statistical analysis of data}

All values were presented as means \pm SD. Statistical significance was analysed by Student t-test and Analysis of Varience (ANOVA) ' $F$ ' test were used for comparison of atherosclerotic CHD in cases groups and controls. The level of significance was set at $P<0.005$.

\section{Results}

The clinical characteristics of CHD patients and control subjects are presented in Table 1. In our study, the markers such as copper and haematological marker such as platelet counts were significantly decreased in CHD patients as compared to controls $(P<0.005)$ (Table 1$)$ whereas when Statistical analysis were carried out by applying student's T test (unpaired) and correlation between variables were studied by using Pearson's correlation coefficient test for comparison of atherosclerotic CHD in cases and controls. The level of significance was set at $P<0.05$.

The normal copper levels vary from 0.70 to $1.55 \mathrm{mg} / \mathrm{dl}$. The levels of copper in the age and sex matched apparently 
healthy controls were between $0.16-2.80 \mathrm{mg} / \mathrm{dl}$ and mean value was $0.98 \pm 0.52$. Range of copper for CHD cases was 0.03 to 1.52 and mean value of copper for CHD cases was $0.83 \pm 0.34$, which is not much less than the mean value in control subjects. In the present study 39 patients of coronary heart disease had copper levels between 1.01 and $1.35 \mathrm{mg} / \mathrm{dl}$ whereas in other 61 patients copper levels were between 0.03 to $0.99 \mathrm{mg} / \mathrm{dl}$.

The characteristics of the enrolled 100 patients with atherosclerotic CHD and 100 controls after age and gender matching are depicted in Table 2. The age at the time of diagnosis of atherosclerotic CHD was distributed between 40-50years, 51-60 years and 61-70 years, 97 out of 200 subjects belong to the age group of 51-60 years followed by 54 out of 200 from age group of 40-50 years and 47 out of 200 from age group of $61-70$ years and the female to male ratio was nearly 8.5:1 (89.50 per cent vs. 10.50 per cent). Compared with the controls, patients with atherosclerotic $\mathrm{CHD}$ versus controls were more likely to have all the comorbidities we had considered including hypertension (34.0 per cent vs. 4.0 per cent) and obesity (34.0 per cent vs. 5.0 per cent).

The reference range for clotting time in healthy individuals is $4-8$ minutes (Lee \& white Method). In the present study, there is a no significant $(p=0.089)$ change found among the atherosclerotic coronary heart disease cases compared to the controls (Table 1).

The reference value of BT in adults is 1-7 minutes (Ivy Method). In the present study, there is no significant $(p=0.628)$ change found among the atherosclerotic coronary heart disease subjects compared to the controls (Figure 1 and Table 1).

Levels of platelet count in 25 patients of coronary heart disease were between 0.75000 and $2.0700010^{5} / \mathrm{mm}^{3}$ whereas in other 75 patients, platelet counts were between 2.01000 to $4.1800010^{5} / \mathrm{mm}^{3}$. The reference value of copper in adults is $0.70-1.55 \mathrm{mg} / \mathrm{dl}$ and a platelet count in adults is 1.50000 to $4.5000010^{5} / \mathrm{mm}^{3}$.

Post hoc analysis of platelet count and copper level by Pearson correlation coefficient of $\mathrm{CHD}$ cases and control subjects (Table 3 ) revealed that, there were significant positive correlation observed between the copper and platelet count levels in CHD cases (Figure 3A), on contrary there was negative correlation observed in copper levels among the control subjects (Figure $3 \mathrm{~B}$ ), which was not statistically significant.

\section{Discussion}

Strong role of platelet in the course of acute coronary syndromes is evident in myocardial infarction, unstable angina and sudden cardiac death as their association with alterations in the haemostatic system, especially with alterations in platelet behaviour. ${ }^{7}$ Alteration in platelet aggregability has been demonstrated as an independent risk factor for a prospective coronary event. The responsiveness and activity of platelets towards aggregating stimuli will increase in subjects who will undergo cardiovascular events. ${ }^{8}$ The impact of platelet behaviour in CHD events is easily understood by the therapeutic efficacy of inhibition of platelet function with aspirin.

In the present study, we demonstrated that there is decreased in platelet count and copper levels between control subjects and those with atherosclerotic CHD disease. Furthermore the post doc analysis shows significantly positive correlation in copper and platelet count levels in CHD cases, on contrary, negative correlated was observed among the level of copper and platelet counts, in the control subjects which was not very significant.

Although the correlation between the copper and platelet count were weak but it again highlighted the fact that copper plays an important role myocardium metabolism associated with platelet aggregation. Deficiency of these elements like $\mathrm{Cu}$ causes cardiomyopathy as a result of decrease levels of vital enzymes. ${ }^{9}$ This observation directed to hypothesis that CHD is predominantly a disease caused by disturbance with respect to copper metabolism. ${ }^{9}$ Furthermore, In the microcirculation, it has been observed that copper-dependent processes are intricately involved in the interactions between endothelial cells and adjacent blood components and vascular smooth muscle. Copper deficiency prolongs haemostasis, inhibits NO-dependent dilation and increases mast cell-mediated macromolecular leakage.

In the present study, there was significant reduction $(p<0.001)$ in platelet counts among the atherosclerotic subjects compared to the controls (Table 1, Figure 2). The findings of our study are contradicting with the study of Pizzulli A. Yang, et al. who showed the decrease number of platelet counts in case of atherosclerotic CHD. ${ }^{10}$ Decreased platelet counts in cases compared to control may be due to increased aggregation of platelets. Decreased level of copper along with decreased level of platelet counts indicate towards the crucial role of copper in platelet 
aggregation. ${ }^{10}$ Copper can influence platelet aggregation via primarily altered prostanoid production. During copper deficiency, platelet thromboxane production would be increased. Since thromboxane potentiates the release of the platelet granules contents, including the aggregating agent, $A D P$, enhancement of the amount of thromboxane would likely be a pro- aggregator effect of copper deficiency. ${ }^{11}$

The second possible mechanism- for the increased platelets aggregation could be related to release of ADP from the dense granules secretion ${ }^{12}$ which would increase the availability of ADP. ADP stimulates platelet alpha- granule secretion and induces exposure of fibrinogen binding between platelets. The in vivo platelet aggregation along with copper deficiency is likely a result of the combination of these enhanced aggregator stimuli.

Thus the low copper levels may be an additional contributing risk factor for myocardial infarction amongst patients with atherosclerosis or patients with low copper levels may progress to myocardial infarction more rapidly than those with normal copper levels. Subjects having diabetes mellitus, hypertension and family history of CHD, supplementation of copper in diet may delay development of atherosclerotic CHD and copper can be used as an addition marker for risk assessment and also as strategic indicators for prevention of reoccurrence of $\mathrm{CHD}$. We recommend copper should be included in routine investigation. It shall not be unnecessary economical burden on the subjects but mortality rate is expected to be decreased.

\section{Limitations of the Study}

This study is limited by the non availability of data on Smoking pattern, family history of CHD and physical activity of study subjects. Furthermore, the small sample size and unequal distribution in the cases groups could have influenced the power of the study to detect significant results.

\section{Conclusion}

The results of aforementioned tests lead to observations that the levels of Copper and platelet counts in this study are less in CHD patients especially in group III cases as compared to other group and control subjects/ reference range. Decreased platelet count and copper levels in cases suggest their important role in atherogenesis which can be detected much earlier than the symptoms arise. This approach can be utilized as preventive measure for delaying the onset of atherosclerosis and also as prognostic marker in CHD cases.

\section{References}

1. Gaziano T, Reddy KS, Paccaud F, et al. Cardiovascular Disease. In: Jamison DT, Breman JG, Measham AR, et al., editors. Disease Control Priorities in Developing Countries. 2nd edition. Washington (DC): The International Bank for Reconstruction and Development/ The World Bank; 2006. Chapter 33. Copublished by Oxford University Press, New York.

2. Gill D, Monori G, Georgakis MK, et al. Genetically Determined Platelet Count and Risk of Cardiovascular Disease. Mendelian Randomization Study. Arterioscler Thromb Vasc Biol. 2018;38:2862-2869.

3. Jlamb D, Reeves GL, Taylor A, et al. Dietary copper supplementation reduces atherosclerosis in the cholesterol-fed rabbit. Atherosclerosis. 1999;146(1):3343.

4. Dacie JV, Lewis SM. Practical Haematology. Capillary action. 3rd Edition Page No.246 and 247.

5. Bauer JD, Ackermann PG, Toro G. Bray clinical laboratory method. (Rees -Ecker Method): p no. 124.

6. Dacie JV, Lewis SM. Practical Haematology. Capillary action. 3rd Edition Page No. 211-212

7. Tofler GH, Brezinski D, Schafer Al, et al. Concurrent morning increase in platelet aggregability and the risk of MI and sudden cardiac death. N Engl J Med. 1987;316:1514-18.

8. Thaulow E, Erikssen J, Sandvik L, et al. Blood platelet count and function are related to total and cardiovascular death in apparently healthy men. Circulation. 1991;84:613-17.

9. Kosar F, Sahin I, Taskapan C, et al. Trace element $(\mathrm{Zn}, \mathrm{Se}, \mathrm{Cu})$ status in heart failure: Anadolu Kardiyol Derg. 2006;6;216-20.

10. Pizzulli L, Yang A, Martin JF, et al. Changes in platelet size and count in unstable angina compared to stable angina or non-cardiac chest pain. Eur Heart J. 1998;19:80-84.

11. Nording HM, Seizer $P$, Langer HF. Platelets in Inflammation and Atherogenesis. Front Immunology. 2015;6:98:1-10.

12. Hou Y, Carrim N, Wang Y, et al. Platelets in haemostasis and thrombosis: Novel mechanisms of fibrinogenindependent platelet aggregation and fibronectinmediated protein wave of haemostasis. J Biomed Res. 2015;29(6):437-444.

\section{ACKNOWLEDGEMENTS}

Authors would like to thank Pathology Department of J N. Medical College, and Heart Foundation of KLES Dr. Prabhakar Kore Hospital \& MRC, Belgaum Karnataka, India. 


\section{PEER REVIEW}

Not commissioned. Externally peer reviewed.

\section{CONFLICTS OF INTEREST}

The authors declare that they have no competing interests.

\section{FUNDING}

None

\section{ETHICS COMMITTEE APPROVAL}

This project was approved by Institutional Ethical Committee of Jawaharlal Nehru Medical College, KLE University, Belgaum, Karnataka, India. 
Table 1: Haematological markers\& Copper levels in controls and atherosclerotic coronary heart disease cases ( $n=200$ )

\begin{tabular}{|c|c|c|c|c|c|c|c|c|c|}
\hline \multirow{2}{*}{ Markers } & \multicolumn{3}{|c|}{ Control } & \multicolumn{3}{c|}{ Cases } & \multirow{2}{*}{ ' $\mathrm{p}^{\prime}$ value } & \multirow{2}{*}{ DF } \\
\cline { 2 - 10 } & Percentage & SD & Range & Percentage & SD & Range & & 0.169 & 198 \\
\hline CT (min) & 5.62 & 0.72 & $4.3-7$ & 5.45 & 0.69 & $3-6.3$ & 1.382 & 0.59 \\
\hline BT (min) & 2.66 & 0.57 & $02-4$ & 2.61 & 0.49 & $1.3-3.3$ & 0.54 & 0.59 & 198 \\
\hline $\begin{array}{c}\text { PLC } \\
\left(10^{5} / \mathrm{mm}^{3}\right)\end{array}$ & 2.82660 & 0.41991 & $\begin{array}{c}1.9600- \\
3.6100\end{array}$ & 2.53240 & 0.75307 & $\begin{array}{c}0.75000- \\
4.18000\end{array}$ & 2.854 & 0.005 & 198 \\
\hline $\mathrm{Cu} \mathrm{mg} / \mathrm{dl}$ & 0.98 & 0.52 & $\begin{array}{c}0.16- \\
2.80\end{array}$ & 0.83 & 0.34 & $\begin{array}{c}0.03- \\
1.52\end{array}$ & 2.377 & 0.018 & 198 \\
\hline
\end{tabular}

Figure 1: Histogram of demographic profile of 200 subjects (100 control and 100 cases)

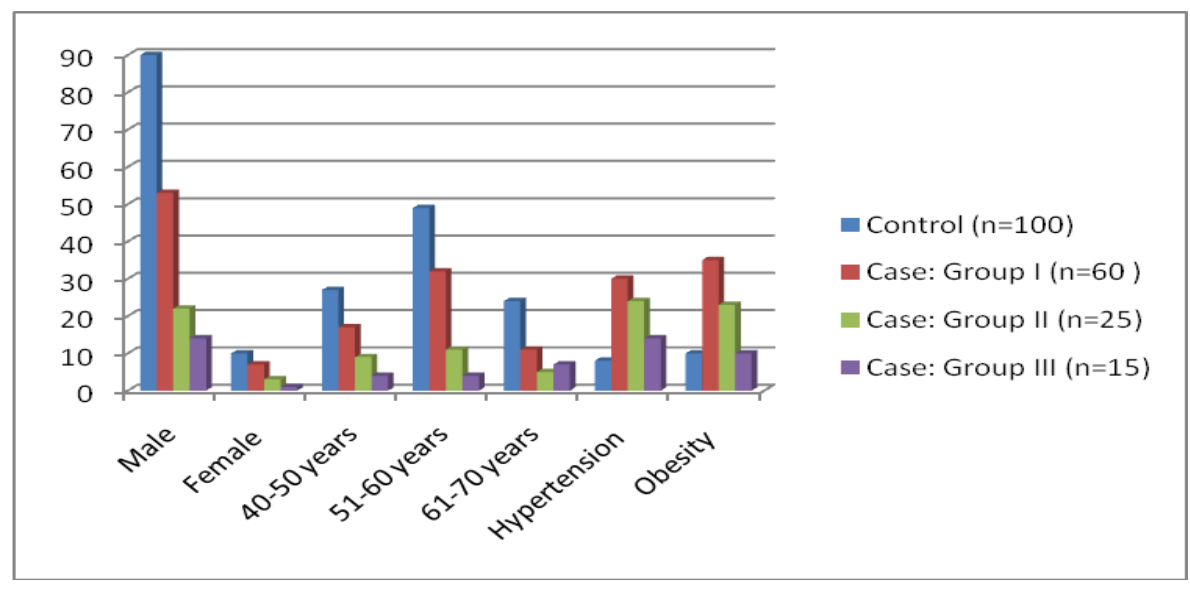

Table 2: Demographic profile of controls and atherosclerotic coronary heart disease cases $(n=200)$.

\begin{tabular}{|l|l|l|l|l|}
\hline & Control $(\mathrm{n}=100)$ & Case: Group I $(\mathrm{n}=60)$ & Case: Group II $(\mathrm{n}=25)$ & Case: Group III $(\mathrm{n}=15)$ \\
\hline Male & 90 & 53 & 22 & 14 \\
\hline Female & 10 & 07 & 03 & 01 \\
\hline $40-50$ years & 27 & 17 & 09 & 04 \\
\hline $51-60$ years & 49 & 32 & 11 & 04 \\
\hline $61-70$ years & 24 & 11 & 05 & 07 \\
\hline Hypertension & 08 & 30 & 24 & 14 \\
\hline Obesity & 10 & 35 & 23 & 10 \\
\hline
\end{tabular}

Figure 2: Histogram of mean haematological markers in controls and cases

\section{-Series1 Series2}

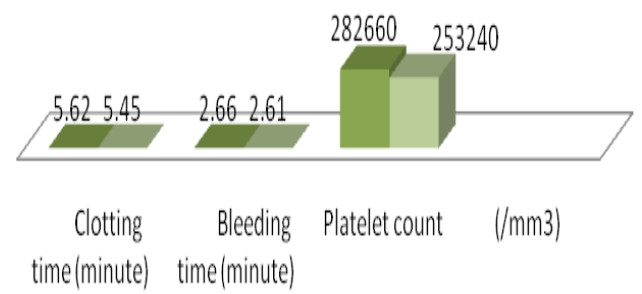


Table 3: Correlation among Platelet count \& Copper levels in controls and atherosclerotic coronary heart disease groups $(n=200)$

\begin{tabular}{|c|c|c|c|c|c|c|c|}
\hline & \multicolumn{2}{|c|}{$\mathrm{PC}\left(10^{5} / \mathrm{mm}^{3}\right)$} & \multicolumn{2}{|c|}{ Copper (mg/dl) } & \multirow[t]{2}{*}{$R$ value } & \multirow[t]{2}{*}{$P$ value } & \multirow{2}{*}{$\begin{array}{l}\mathbf{R}^{2} \text { coefficient of } \\
\text { determination }\end{array}$} \\
\hline & Percentage & SD & Percentage & SD & & & \\
\hline Control $(n=100)$ & 2.86780 & 0.41991 & 0.98 & 0.52 & -0.0784 & 0.2191 & 0.0061 \\
\hline Case(n=100) & 2.53240 & 0.75307 & 0.83 & 0.34 & 0.2281 & 0.0112 & 0.052 \\
\hline
\end{tabular}

Figure 3A: Negative Correlation among Platelet count \& Copper levels in control groups ( $\mathrm{n}=\mathbf{2 0 0})$

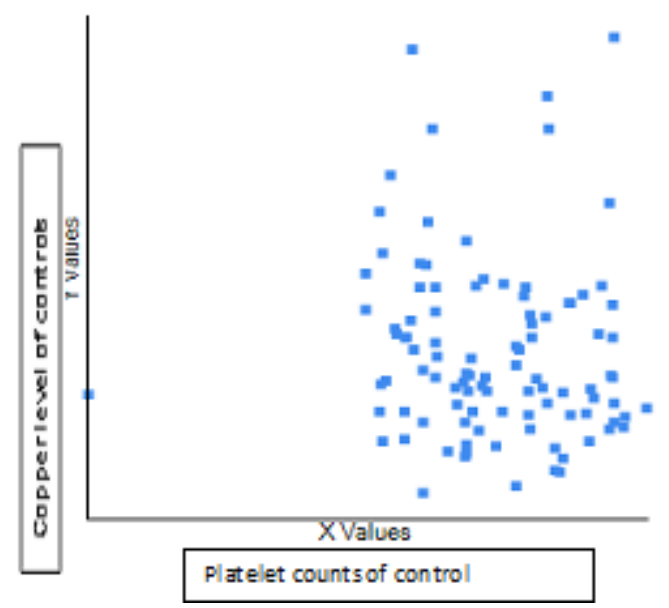

Figure 3B: Positive Correlation among Platelet count \& Copper levels in atherosclerotic coronary heart disease groups $(n=200)$

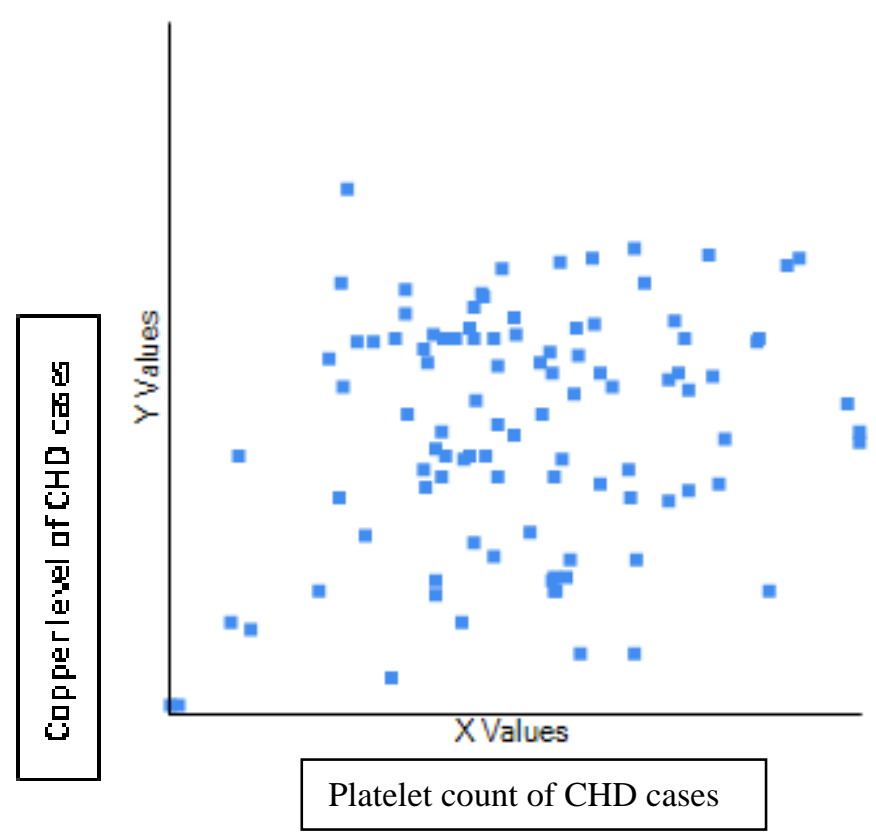

